\title{
On-Surface Synthesis and Characterization of
}

\section{9-Atom Wide Armchair Graphene Nanoribbons}

Leopold Talirz ${ }^{1, \dot{\dagger},+}$, Hajo Söde ${ }^{1, \dot{+}}$, Tim Dumslaff ${ }^{2,+}$, Shiyong Wang ${ }^{1}$, Juan Ramon Sanchez-

Valencia $^{l}$, Jia Liu ${ }^{l}$, Prashant Shinde ${ }^{1}$, Carlo A. Pignedoli ${ }^{1,3}$, Liangbo Liang ${ }^{4,5}$, Vincent Meunier ${ }^{4}$,

Nicholas C.Plumb ${ }^{6}$, Ming Shi ${ }^{6}$, Xinliang Feng ${ }^{7}$, Akimitsu Narita $^{2}$, Klaus Müllen $^{2}$, Roman Fasel ${ }^{1,8}$, Pascal Ruffieux ${ }^{1, *}$

${ }^{1}$ Empa, Swiss Federal Laboratories for Materials Science and Technology, nanotech@surfaces Laboratory, 8600 Dübendorf, Switzerland

${ }^{2}$ Max Planck Institute for Polymer Research, 55128 Mainz, Germany

${ }^{3}$ NCCR MARVEL, Empa, Swiss Federal Laboratories for Materials Science and Technology, 8600 Dübendorf, Switzerland

${ }^{4}$ Department of Physics, Applied Physics, and Astronomy, Rensselaer Polytechnic Institute, Troy, 12180 NY, USA

${ }^{5}$ Center for Nanophase Materials Sciences, Oak Ridge National Laboratory, Oak Ridge, 37831 Tennessee, USA

${ }^{6}$ Swiss Light Source, Paul Scherrer Institute, 5232 Villigen, Switzerland

${ }^{7}$ Center for Advancing Electronics Dresden \& Department of Chemistry and Food Chemistry, Technische Universität Dresden, 01062 Dresden, Germany 
${ }^{8}$ Department of Chemistry and Biochemistry, University of Bern, 3012 Bern, Switzerland

KEYWORDS graphene nanoribbons, bottom-up synthesis, scanning tunneling spectroscopy, Raman spectroscopy, on-surface chemistry

\section{ABSTRACT}

The bottom-up approach to synthesize graphene nanoribbons strives not only to introduce a band gap into the electronic structure of graphene, but also to accurately tune its value by designing both width and edge structure of the ribbons with atomic precision. We report the synthesis of an armchair graphene nanoribbon with a width of 9 carbon atoms on $\mathrm{Au}(111)$ through surfaceassisted aryl-aryl coupling and subsequent cyclodehydrogenation of a properly chosen molecular precursor. By combining high-resolution atomic force microscopy, scanning tunneling microscopy and Raman spectroscopy, we demonstrate that the atomic structure of the fabricated ribbons is exactly as designed. Angle-resolved photoemission spectroscopy and Fouriertransformed scanning tunneling spectroscopy reveal an electronic band gap of $1.4 \mathrm{eV}$ and effective masses of $\approx 0.1 m_{e}$ for both electrons and holes, constituting a substantial improvement over previous efforts towards the development of transistor applications. We use $a b$ initio calculations to gain insight into the dependence of the Raman spectra on excitation wavelength, as well as to rationalize the symmetry-dependent contribution of the ribbons' electronic states to the tunneling current. We propose a simple rule for the visibility of frontier electronic bands of armchair graphene nanoribbons in scanning tunneling spectroscopy. 
The discovery of the field effect in graphene in 2004, together with the high mobility of its charge carriers ${ }^{1}$, caused excitement in the electronics industry and has enabled the fabrication of prototype transistors based on graphene that operate at frequencies beyond $400 \mathrm{GHz}^{2}$. However, since graphene lacks an electronic band gap, graphene-based transistors do not completely turn off at room temperature. This is a major obstacle to applications both in digital electronics and in high-frequency signal amplification., ${ }^{3,4}$ One way to open a band gap is to cut graphene into narrow ribbons. Quantum confinement offers the exciting possibility of tuning the gap to just the right value for the intended application by choosing the width of the graphene nanoribbons (GNRs) accordingly. The resulting band gap $\Delta$ is then approximately inversely proportional to the ribbons' width, and band gaps suitable for room temperature applications are reached for widths in the low-nm regime. ${ }^{5,6}$ At this scale, however, the width and edge structure of the GNRs need to be controlled with atomic precision in order to produce predictable transport properties. ${ }^{7,8}$

These stringent requirements pose a major challenge for traditional top-down structuring, which offers limited resolution and lacks control over the chemical passivation of the edges. ${ }^{9}$ The requirements can, however, be met by switching to a bottom-up fabrication strategy that relies on the coupling and subsequent cyclodehydrogenation of suitable precursor molecules on a metal surface..$^{10}$ In this approach, the molecular precursor defines the width and edge structure of the resulting GNRs down to the single atom, thus providing ultimate control over the band gap by design. The challenge is to synthesize suitable precursor molecules, to find optimal reaction parameters for their polymerization and, eventually, to tune the band gap of the GNRs to technologically desired values. So far, much work has been directed towards GNRs with (monohydrogenated) armchair edges, ${ }^{10-13}$ which are more chemically stable than those with zigzag edges. ${ }^{14}$ Armchair graphene nanoribbons (AGNRs) are labeled by the number $N$ of carbon 
atoms across their width, as sketched in Figure 1. $N$-AGNRs of width $N=5,{ }^{12,15} N=7,{ }^{10}$ and $N=13^{13}$ have been obtained using this strategy. 6-AGNRs have been obtained via cross-dehydrogenative coupling of pre-aligned poly( $p$-phenylene) chains, although without selective control over their width. ${ }^{16}$

For GNRs with widths in the low-nm regime, the simple relation $\Delta \propto 1 / w$ between band gap $\Delta$ and width $w$ is predicted to break down. ${ }^{6,17}$ Armchair GNRs split into three distinct families, based on whether their width $N$ is of the form $N=3 m-1$ (small- $\Delta$ ), $3 m$ (medium- $\Delta$ ) or $3 m+1$ (large- $\Delta)$, where $m$ is an integer. Within each family, $\Delta$ increases monotonically as the GNR width is decreased, but stepping through the widths atom by atom corresponds to alternating between families and gives rise to non-monotonous variation in $\Delta$. The best-characterized AGNR so far is the 7-AGNR, which belongs to the large- $\Delta$ family. Using scanning tunneling spectroscopy on $\mathrm{Au}(111)$, a band gap of $\Delta=2.4 \mathrm{eV}$ and effective masses of $\approx 0.4 m_{e}$ for valence and conduction bands have been determined, where $m_{e}$ denotes the free electron mass. ${ }^{18}$ Given that the 9-AGNR is both wider than the 7-AGNR and belongs to the medium- $\Delta$ family, a significant reduction in both band gap and effective masses is expected, making this GNR an interesting candidate for transistor applications.

In the following, we describe the synthesis of the armchair GNR with width $N=9$ (9-AGNR) on the Au(111) surface using a small molecular precursor that enters the GNR in two different orientations. We characterize the GNR's atomic and electronic structure, finding a band gap of $\Delta=1.4 \mathrm{eV}$ and low effective masses of $\approx 0.1 \mathrm{~m}_{\mathrm{e}}$ for both valence and conduction band.

\section{Results and Discussion}


Synthesis. The on-surface synthesis of long, low-defect graphene nanoribbons requires the molecular precursors to be able to approach each other on the surface without steric hindrance. Furthermore, small precursors simplify the on-surface synthesis by limiting the number of possible conformations the molecule can adapt on the surface. The dibromo-o-terphenyl precursor 7 (Figure 1) has been designed with these criteria in mind.

The synthesis of $o$-terphenyls substituted by two halogens, especially at the required positions, is not trivial and has rarely been reported. In contrast to the very recent work by Li et al. ${ }^{19}$ who start from 1,2-dibromobenzene, we adopted a different, efficient synthetic strategy outlined in Figure 1a (details on p. S2-S5). The most feasible route was deemed to start from 2,5dibromoaniline $\mathbf{1}$, which was easily converted to the respective (hydroxyimino)acetamide $\mathbf{3}^{20}$ Cyclization with sulfuric acid to 4 and subsequent ring opening under basic conditions with hydrogen peroxide provided 2-amino-3,6-dibromobenzoic acid 5 . Treatment with nitrite led to an aryne intermediate that could be trapped in situ with iodine, yielding 1,4-dibromo-2,3diiodobenzene $6{ }^{21}$ Since iodine, while being more reactive than bromine, is more sterically hindered, the key Suzuki-coupling step needed to be performed at relatively low temperature. After multiple recrystallization steps, $3^{\prime}, 6^{\prime}$-dibromo-1,1':2',1"-terphenyl 7 was obtained in moderate yields and high purity, with no detectable traces of contaminants (such as monobrominated species) in ${ }^{1} \mathrm{H}$ NMR and high-resolution mass spectrometry - a crucial prerequisite for the following on-surface synthesis.

Monomer 7 was sublimated onto the $\mathrm{Au}(111)$ surface under ultrahigh vacuum and activated by surface-assisted dehalogenation at temperatures above $150^{\circ} \mathrm{C}$. Extended one-dimensional polymers 8 formed upon annealing to $250^{\circ} \mathrm{C}$, which lets the activated precursor monomers diffuse across the surface. Owing to the atomic structure of the monomers and their confinement 
to the surface, covalent bonds between monomers can only form if they are rotated by $180^{\circ}$ with respect to each other. This selectivity is essential for achieving linear polymers that can be transformed into 9-AGNRs 9 in the next step. Figure 2 a shows scanning tunneling microscopy (STM) images of $\mathbf{8}$ together with a structural model obtained from density functional theory (DFT) calculations of the finite-length polymer adsorbed on the Au(111) surface. The calculations predict the poly( $p$-phenylene) backbone of $\mathbf{8}$ to be almost planar, while the sterically hindered phenyl side groups are rotated out of plane. This is in line with the apparent height of $0.24 \mathrm{~nm}$ determined by STM. The predicted periodicity with a repeat unit of $0.88 \mathrm{~nm}$ along the polymer axis agrees closely with the periodicity of features determined by STM $(0.86 \pm 0.02 \mathrm{~nm})$, confirming that $\mathrm{C}-\mathrm{C}$ bonds were formed between neighboring precursor monomers. The polymers themselves assembled into extended islands, which points to an attractive interaction between the partially interdigitated phenyl rings of neighboring polymers, as has also been observed in the synthesis of chevron-type GNRs. ${ }^{10}$

Upon further annealing to $350^{\circ} \mathrm{C}$, cyclodehydrogenation of $\mathbf{8}$ induced planarization through intramolecular dehydrogenation and led to the formation of the target structure 9. This was observed as a reduced apparent height of $0.17 \mathrm{~nm}$ in STM and a halving of the periodicity along the ribbon axis $(0.43 \pm 0.02 \mathrm{~nm})$, as expected (Fig. $2 \mathrm{~b}, 2 \mathrm{c})$. The atomic structure of the ribbons was studied via non-contact atomic force microscopy (nc-AFM) using a CO-functionalized tip. ${ }^{22}$ Figure $2 \mathrm{~d}$ reveals the intraribbon bond configuration and directly confirms that the target width of 9 carbon atoms was selectively achieved. We note that the flexibility of the CO tip results in geometric distortions of the AFM image near the GNR edge that make it difficult to accurately determine the individual bond lengths (see p. S6 for details). Nevertheless, the planarity of 9 proves that the edge carbon atoms remained passivated by single hydrogen atoms as expected 
from the design of the precursor 7: the formation of edge radicals (no hydrogen) would yield organometallic bonds to the substrate, resulting in a pronounced downward curvature towards the GNR edges, whereas the $s p^{3}$ geometry associated with $\mathrm{H}_{2}$ passivation would be observed as distinct maxima in the nc-AFM frequency shift images. ${ }^{23}$

Mono-hydrogenated edges are found also at the termini of the 9-AGNR, which exhibit the slanted armchair edge expected from precursor 7 (see Fig. 1b and S1). In contrast to the straight zigzag termination, found e.g. in the synthesis of 7-AGNRs with the dibromo-bianthryl precursor $^{10,24}$ or 5-AGNRs with the dibromo-perylene precursor, ${ }^{15}$ the slanted termination of the 9-AGNR is compatible with the Clar formula for extended 9-AGNRs and is therefore not associated with localized unpaired electrons (Fig. S1a).

Furthermore, nc-AFM identified the nature of the most prominent remaining type of defect, which is due to a missing phenyl ring (see Fig. $2 b$ and S2). The high purity of the monomers after multiple recrystallization steps, combined with the fact that no defects of the polymer $\mathbf{8}$ were discernable in STM, strongly suggest that C-C bond scission occurred during the cyclodehydrogenation reaction. This is in line with previous observations for the synthesis of chevron-type GNRs with the tetraphenyl-triphenylene precursor, ${ }^{10}$ indicating a link between this type of defect and the cyclization of multiple flexible phenyl rings against each other.

On a larger scale, the structural quality of the as-grown 9-AGNRs was analyzed by Raman spectroscopy. Figure 3a displays Raman spectra measured with infrared $(785 \mathrm{~nm}, 1.58 \mathrm{eV})$ and green $(532 \mathrm{~nm}, 2.33 \mathrm{eV})$ laser lines and compares them to DFT-based Raman simulations that take the dependence on the excitation wavelength into account. The wavenumbers of both the radial-breathing-like mode (RBLM) at $312 \mathrm{~cm}^{-1}$ and the $G$ peak at $1597 \mathrm{~cm}^{-1}$ are in good agreement with the simulated spectrum. Since the RBLM frequency varies strongly with GNR 
width (Fig. 3b), the presence of the sharp peak at $312 \mathrm{~cm}^{-1}$ provides further evidence for the high width selectivity of the on-surface synthesis.

The experimental Raman spectra exhibit a strong dependence on excitation wavelength, leading in particular to the suppression of the RBLM for excitation with the green laser (2.33 $\mathrm{eV}$ ). The exact opposite behavior has been observed for 7-AGNRs, where the RBLM is resonantly excited using the green laser, ${ }^{10}$ while no response is detected for the infrared laser (1.58 eV, not shown). Tight-binding calculations indicate that the RBLM of GNRs is coherently excited for photon energies near the lowest optical transitions of the respective GNR, ${ }^{25}$ i.e. the observations for the 7-AGNR may be rationalized by noting that $2.3 \mathrm{eV}$ just slightly exceed the optical gap of $2.1 \mathrm{eV}$, while $1.58 \mathrm{eV}$ fall deep into the gap. ${ }^{26}$ According to prior theoretical work $^{27,28}$ and very recent solution-based characterization, ${ }^{19}$ the optical gap of 9-AGNRs is $1.0-1.1$ $\mathrm{eV}$, which fits both the enhanced response of the RBLM to the infrared laser $(1.58 \mathrm{eV})$ and the determined electronic gap of $1.4 \mathrm{eV}$ on $\mathrm{Au}(111)$ (see next section). We note that our Raman simulations are based on the HSE06 hybrid density functional, ${ }^{29}$ which also predicts a gap of 1.1 $\mathrm{eV}$, and confirm that the response of the RBLM is maximal when the excitation energy coincides with the optical gap (see Fig. S5b).

Electronic Structure. The electronic structure of 9-AGNRs 9 was analyzed by angleresolved photoemission spectroscopy (ARPES) and scanning tunneling spectroscopy (Fig. 4). For ARPES, the GNRs were grown on $\mathrm{Au}(788)$, a periodically stepped surface with narrow (111) terraces, forcing them to align parallel to the step edges. ${ }^{30,31}$ One major advantage of ARPES is its momentum resolution, which allows to unambiguously separate the low-energy contributions from the substrate (surface state and s-p bands) from the 9-AGNR contributions, which are centered at $k=1.47 \AA^{-1}$, corresponding to the projection of the graphene $K$ points onto 
the ribbon axis. The angle-resolved spectra in Figure $4 \mathrm{~b}$ show the dispersion of the GNR valence band along the axis of the aligned GNRs. From a two-parameter least squares fit to $E(k)=E_{0} \pm$ $\frac{\hbar^{2}}{2 m^{*}} k^{2}$ (+ for electrons, - for holes) within the $k$-range $\left[-1 \mathrm{~nm}^{-1}, 1 \mathrm{~nm}^{-1}\right]$, we determine the valence band maximum as $E_{0}=-0.25 \pm 0.04 \mathrm{eV}$ and an effective mass of $m^{*}=0.09 \pm 0.02 m_{e}$.

ARPES is limited to the study of occupied electronic states. In order to gain access to both occupied and unoccupied electronic states, we performed scanning tunneling spectroscopy (STS) on individual 9-AGNRs adsorbed on $\mathrm{Au}(111)$. Figure 4a compares $d I / d V$ spectra recorded on the 9-AGNR to spectra recorded on the bare $\mathrm{Au}(111)$ substrate, revealing ribbon-related peaks at $+1.1 \mathrm{~V}$ (empty states) and $-0.3 \mathrm{~V}$ (occupied states). For the one-dimensional 9-AGNRs, we expect van-Hove singularities at the band edges and thus interpret the peak positions as first estimates for the conduction band minimum and the valence band maximum, respectively. It is important to note that the valence band signal at $-0.3 \mathrm{~V}$ is weak in comparison to contributions from the $\mathrm{Au}(111)$ substrate. It could only be resolved at setpoint currents $\gtrsim 10 \mathrm{nA}(V=-0.5 \mathrm{eV})$, indicating a rapid decay of its relative contribution with increasing tip-sample distances, as discussed in detail in the next section of the manuscript. The energetic position of the valence band maximum, however, is in good agreement with the position determined from ARPES. In the following, the edges of valence and conduction band are pinned down more accurately by momentum-resolved STS.

Figure $4 \mathrm{c}$ presents a series of differential conductance spectra recorded along the axis of a 9AGNR, mapping out energy-resolved standing wave patterns, which arise from scattering of the GNR's electronic states at its termini or at defects (for corresponding STM images see Fig. S4). In the Tersoff-Hamann approximation, ${ }^{32}$ the derivative of the tunneling current $d I / d V$ is proportional to the local density of states (LDOS) at the position of the tip. ${ }^{32}$ Figures $4 \mathrm{c}$, showing 
occupied states, and 3e, showing empty states, can thus be interpreted as maps of the local density of states along the GNR and provide access to both the electronic band gap $\Delta$ and the dispersion relation of valence band (VB) and conduction band (CB) near the Fermi energy.

Figures $4 \mathrm{~d}$ and $4 \mathrm{f}$ present the line-by-line Fourier transform of $4 \mathrm{c}$ and $4 \mathrm{e}$ (for a detailed description of the procedure see ${ }^{18}$ ). The Fourier-transformed LDOS clearly reveals the dispersion of valence and conduction bands with the wave vector $k=q / 2$, while at higher bias voltages the energetic overlap of multiple bands makes it difficult to discern their contributions. Band edges and effective masses for valence and conduction band were determined by a twoparameter parabolic fit as before and are listed in Table 1, where uncertainties related to the fit as well as to the choice of the real-space window for the Fourier transform were incorporated into the error bars. Parameters determined for the valence band were found to agree with those based on ARPES within error bars.

The key results are the low effective mass $m^{*} \approx 0.1 m_{e}$ for both electrons and holes and the low band gap of $\Delta=1.4 \mathrm{eV}$ for the 9 -AGNR on $\mathrm{Au}(111)$. This constitutes a substantial improvement over $m^{*}>0.2 m_{e}$, and $\Delta=2.4 \mathrm{eV}$ for the 7 -AGNR, ${ }^{18}$ which is the only bottom-up fabricated GNR for which transistor prototypes have been reported so far. ${ }^{33}$ In order to validate these experimental findings further, we computed the effective masses of the free-standing 9-AGNRs within density functional theory using the PBE exchange-correlation functional ${ }^{34}$, yielding $m^{*}=$ $0.10 m_{e}$ for both electrons and holes. Quasiparticle corrections within one-shot $G W\left(G_{0} W_{0}\right)$ yield a $\sim 20 \%$ decrease in effective mass to $m^{*}=0.08 m_{e}$. This is in accordance with previous work on graphene, where many-body corrections to the local density approximation yield a $17 \%$ increase in the Fermi velocity. ${ }^{35}$ In order to estimate the (fundamental) band gap of the 9-AGNRs while adsorbed on $\mathrm{Au}(111)$, we combined the quasiparticle corrections computed for the freestanding 
9-AGNR at the $G_{0} W_{0}$ level with a classical image charge model that takes both the adsorption distance of 9-AGNRs on $\mathrm{Au}(111)$ and their intrinsic polarizability into account. This method yields $\Delta^{G W}+\Delta^{I C}=2.1 \mathrm{eV}-1.0 \mathrm{eV}=1.1 \mathrm{eV}$, in acceptable agreement with the experimental value of $1.4 \mathrm{eV}$ considering the approximations involved, (one-shot $G W$ with plasmon pole model, classical image charge model for the substrate).

\section{Relative Contribution of Bands in STS at Finite Tip-Sample Distance. When} analyzing scanning tunneling spectra, it is important to keep in mind that STS is performed at tip-sample distances of a few Angstroms and thus probes the exponential tails of the electronic states of the GNR. The effect of finite tip-sample distances is illustrated in Figure 5 by means of STS simulations based on density functional theory. Figure 5a shows the Kohn-Sham orbitals of a finite 9-AGNR at the respective band edges. The orbitals have been evaluated once on a plane $0.1 \mathrm{~nm}$ above the GNR (a), corresponding to a short tip-sample distance, and once at a larger distance of $0.4 \mathrm{~nm}$ (b). While the orbital arising from the VB oscillates strongly along and perpendicular to the ribbon axis, the orbital arising from the $\mathrm{CB}$ does not change sign along the ribbon axis. As a consequence, the LDOS associated with the CB decays more slowly than its VB counterpart and is concentrated at the GNR edges. This can be understood by realizing that the effect of moving away from the GNR is approximately that of a Gaussian filter, smearing out positive and negative regions of the wave function (see p. S12-S13 for details). We now follow the same procedure as in experiment, by first calculating the LDOS along the 9-AGNR [(c),(d)] and then taking its Fourier transform [(e),(f)]. In Figure 5e, corresponding to $0.1 \mathrm{~nm}$ tip-sample distance, the edges of frontier bands can be clearly determined from the FT-LDOS. In Figure $5 f$ at $0.4 \mathrm{~nm}$ distance, however, the signal from the VB is missing completely. Note that this problem was addressed experimentally by a tenfold increase in set point current for the 
conductance spectra of the valence states (Fig. 4a), corresponding to a reduction in tip-sample distance of $\approx 0.1 \mathrm{~nm}$.

Similar effects have been observed for the 7-AGNR, but here the roles of conduction and valence band are reversed and it is the conduction band that suffers from weak signal in STS. ${ }^{18,36}$ Such variations in signal strength can lead to wrong assignment of the frontier bands and, hence, a wrong band gap extracted from STS. A systematic prediction of these effects would thus be highly useful in order to guide future experiments. As discussed in the following, this symmetryrelated effect can readily be understood at the tight-binding level, using only pen and paper.

In a single-orbital nearest-neighbor tight-binding picture, the band structure of $N$-AGNRs (of infinite length) can be obtained by $N$ slices of the band structure of graphene in reciprocal space and analytical expressions are available for the corresponding wave functions. ${ }^{37,38}$ Figure $6 \mathrm{a}$ shows these slices for the 9-AGNR, labeled from $r=1$ for the slice closest to $\Gamma$ to $r=9$. For vanishing longitudinal crystal wave vector $k$ (dotted line in Fig. 6a and 6b), and thus both at the top of the valence band and the bottom of the conduction band, the wave functions share the periodicity of the underlying atomic lattice. In this case, the relative phase $\phi$ of the wave function on two directly adjacent carbon atoms along the armchair direction becomes particularly simple: it may either be +1 (in phase) or -1 (out of phase), as derived on p. S12-S13. Specifically, bands arising from a slice $r$ have phase $\phi=-s$, if $\frac{r}{N+1}<\frac{2}{3}$ (slice located between $\Gamma$ and $\mathrm{K}$ ), and $\phi=s$, if $\frac{r}{N+1}>\frac{2}{3}$, where $s=+1$ for the unoccupied states and $s=-1$ for the occupied states.

Figure $6 \mathrm{~b}$ illustrates the results for the 9-AGNR. Here, valence and conduction band arise from the slice labeled by $r=7$, yielding $\phi=-1$ for the valence band and $\phi=+1$ for the conduction 
band. This implies a suppression of the signal from the valence band in STS (near $k=0$ ), in agreement with the DFT-based analysis shown in Figure 5. The above reasoning applies to all $\pi$ bands of $N$-AGNRs of all families, except for those bands passing directly through the Dirac point $K$. In particular, weak signals are expected for the valence band in the medium- $\Delta$ family $(N=3 m)$ and for the conduction band in the large- $\Delta$ family $(N=3 m+1)$. The effect can be counteracted by a reduction in tip-sample distance within experimental constraints.

\section{Conclusions}

We have presented the synthesis of 9-atom wide armchair graphene nanoribbons through surface-assisted aryl-aryl coupling and cyclodehydrogenation of a small halogen-substituted precursor molecule on $\mathrm{Au}(111)$. Scanning probe microscopy and Raman spectroscopy reveal atomic precision with respect to width and edge passivation of the 9-AGNRs, demonstrating the fully selective conversion of the precursor molecules. In line with theoretical predictions of their electronic properties, a low band gap of $1.4 \mathrm{eV}$ and low effective masses of $0.1 m_{e}$ for electrons and holes are found for the metal-adsorbed 9-AGNRs, making them an interesting material for room-temperature electronic and optoelectronic switching devices. Furthermore, we have analyzed the band-dependent vacuum-decay of the AGNR wave functions at the level of density functional theory and tight binding. We find that in scanning tunneling spectroscopy studies of $N$-AGNRs, weak signals are expected for the valence band in the $N=3 m$ family and for the conduction band in the $N=3 m+1$ family. In these cases, signal may be recovered by a reduction in tip-sample distance. 


\section{Methods}

Monomer Synthesis. Detailed descriptions of reaction steps and characterization methods are given in the supporting information on p. S2-S5.

\section{On-surface synthesis and characterization}

On-surface synthesis steps were performed in an ultra-high vacuum (UHV) chamber, directly connected to a low-temperature scanning tunneling microscope (Scienta Omicron) operated at $5 \mathrm{~K}$. The $\mathrm{Au}(111)$ substrate was prepared by repeated sputtering $\left(\mathrm{Ar}^{+}\right.$ions, $\left.1 \mathrm{keV}\right)$ and annealing (720 K) cycles. Molecular precursors were thermally evaporated onto the clean $\mathrm{Au}(111)$ substrate from a quartz crucible heated to $390 \mathrm{~K}$, resulting in a deposition rate of $0.1 \mathrm{~nm} / \mathrm{min}$. STM images were recorded in constant-current mode and $d I / d V$ spectra were obtained using a lock-in amplifier by modulating the sample bias with $20 \mathrm{mV}$ and a frequency of $451 \mathrm{~Hz}$. NCAFM measurements were performed with a CO-functionalized tungsten tip attached to a tuning fork sensor ${ }^{39}$. The sensor was driven at its resonance frequency $(23.570 \mathrm{kHz})$ with constant amplitude. Unpolarized micro-Raman spectra were acquired in a Bruker Senterra instrument using two different lasers, green at $532 \mathrm{~nm}$ and near-infrared at $785 \mathrm{~nm}$ with 20 and $100 \mathrm{~mW}$, respectively, both with a spectral resolution of $3-5 \mathrm{~cm}^{-1}$. The measurements were performed with a 50x magnifying objective with numerical aperture 0.75 . ARPES measurements were performed at the Surface and Interface Spectroscopy (SIS) beamline of the Swiss Light Source on samples prepared in the low-temperature STM chamber on the $\mathrm{Au}(788)$ surface, a stepped $\mathrm{Au}(111)$ surfaces with terraces of $\approx 4 \mathrm{~nm}$ width that unidirectionally align the 9-AGNRs along the step edges. Prior to photoemission experiments, volatile contaminants originating from exposure to 
air were desorbed by heating the sample to $450 \mathrm{~K}$ in a UHV chamber connected to the photoemission setup.

Computational Procedures. Density functional theory (DFT) calculations of 9-AGNRs in vacuum were performed using the PBE generalized gradient approximation to the exchangecorrelation functional ${ }^{34}$. Structures were relaxed until the forces acting on the atoms were below $3 \mathrm{meV} / \AA$. For finite ribbons (and the polymer shown in Fig. 2a) we used the CP2K code ${ }^{40}$, which expands the wave functions on an atom-centered Gaussian-type basis set. After extrapolating the electronic states into the vacuum region ${ }^{41}$ (details on p. S12-S13), STS simulations were performed in the Tersoff-Hamann approximation ${ }^{32}$ on a plane parallel to the planar GNR, using a Lorentzian broadening of full-width $150 \mathrm{meV}$ at half maximum. Band structure and effective masses of the infinite 9-AGNR were calculated with the Quantum ESPRESSO package ${ }^{42}$ using optimized norm-conserving pseudopotentials ${ }^{43,44}$, a cutoff of 80 Ry for the plane wave basis, and a grid of $128 k$-points in the first Brillouin zone.

For Raman calculations, the atomic and electronic structure of the 9-AGNRs were recomputed using the local-density approximation in the projector-augmented wave formalism as implemented in the VASP package ${ }^{45}$. Phonon spectra were computed via the finite-difference scheme in a threefold supercell with atomic displacements $\delta=0.03 \AA$, using the PHONON software to construct the dynamical matrix ${ }^{46}$. Dielectric tensors were computed for positive and negative displacements in the unit cell, switching to the HSE06 hybrid functional ${ }^{29}$ and using PHONON to compute their derivatives. Following the experimental setup, the Raman intensity was calculated for laser energies $1.58 \mathrm{eV}$ (infrared) and $2.33 \mathrm{eV}$ (green) at normal incidence, averaging over all in-plane polarization directions (more details on p. S8-S11). 
Quasiparticle corrections in the $G_{0} W_{0}$ approximation were computed using the BerkeleyGW package. ${ }^{47,48}$ The static dielectric matrix was calculated using a rectangular Coulomb-cutoff along the aperiodic directions ${ }^{49}$ and extended to finite frequencies by the generalized plasmon pole model ${ }^{47}$ In the calculation of the self-energy, the static remainder approach was used to speed up the convergence with respect to the number of empty bands (more details on p. S11-S12).$^{50}$

\section{a}
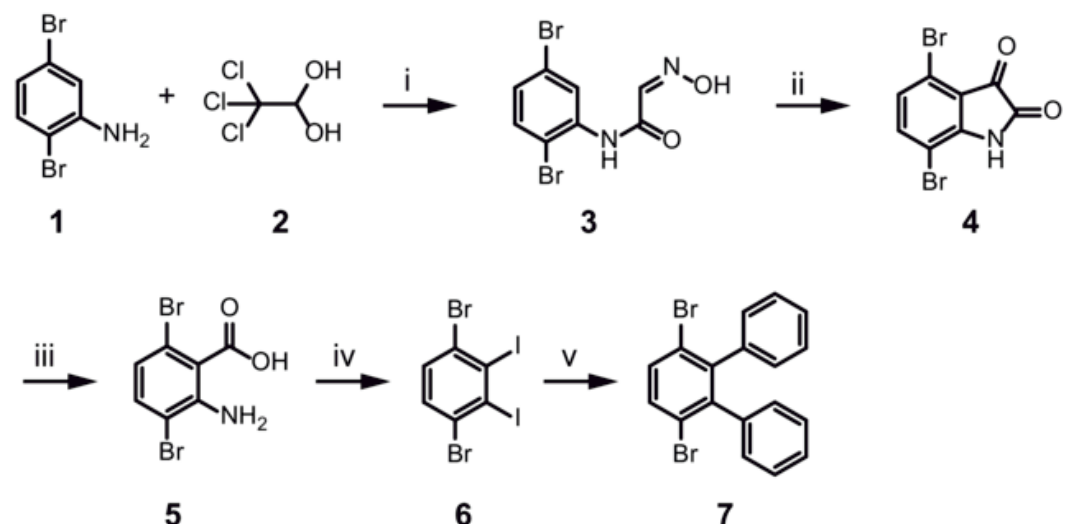

b

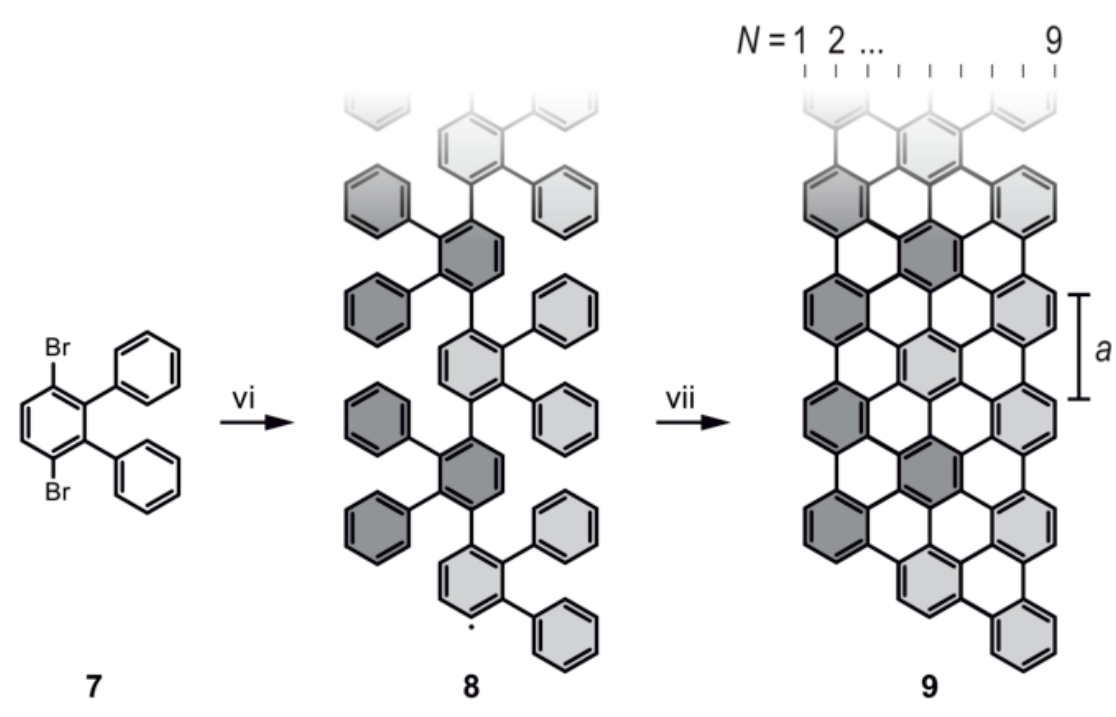

Figure 1. (a) Synthetic route towards 3',6'-dibromo-1,1':2',1"-terphenyl 7. (b) On-surface synthesis of 9-atom wide armchair graphene nanoribbon 9 via surface-assisted dehalogenation and polymerization of monomer $\mathbf{7}$, followed by cyclodehydrogenation of polymer $\mathbf{8}$. Reagents 
and conditions: (i) hydroxylamine hydrochloride, $\mathrm{H}_{2} \mathrm{O}$, EtOH, $80{ }^{\circ} \mathrm{C}, 12 \mathrm{~h}$; (ii) $\mathrm{H}_{2} \mathrm{SO}_{4}, 100{ }^{\circ} \mathrm{C}, 30$ min; (iii) $\mathrm{NaOH}, \mathrm{H}_{2} \mathrm{O}_{2}, 50{ }^{\circ} \mathrm{C}, 30 \mathrm{~min}$, then $\mathrm{HCl}$, rt; (iv) isoamylnitrite, $\mathrm{I}_{2}, 1$,2-dichloroethane, dioxane, 1h, reflux; (v) phenylboronic acid, $\mathrm{Na}_{2} \mathrm{CO}_{3}, \mathrm{Pd}\left(\mathrm{PPh}_{3}\right)_{4}, \mathrm{H}_{2} \mathrm{O}$, dioxane, $80{ }^{\circ} \mathrm{C}, 2 \mathrm{~d}$; (vi) sublimation onto $\mathrm{Au}(111)$ held at $250{ }^{\circ} \mathrm{C}$, then annealing at $250{ }^{\circ} \mathrm{C}, 10 \mathrm{~min}$; (vii) annealing at $350{ }^{\circ} \mathrm{C}, 10 \mathrm{~min}$.
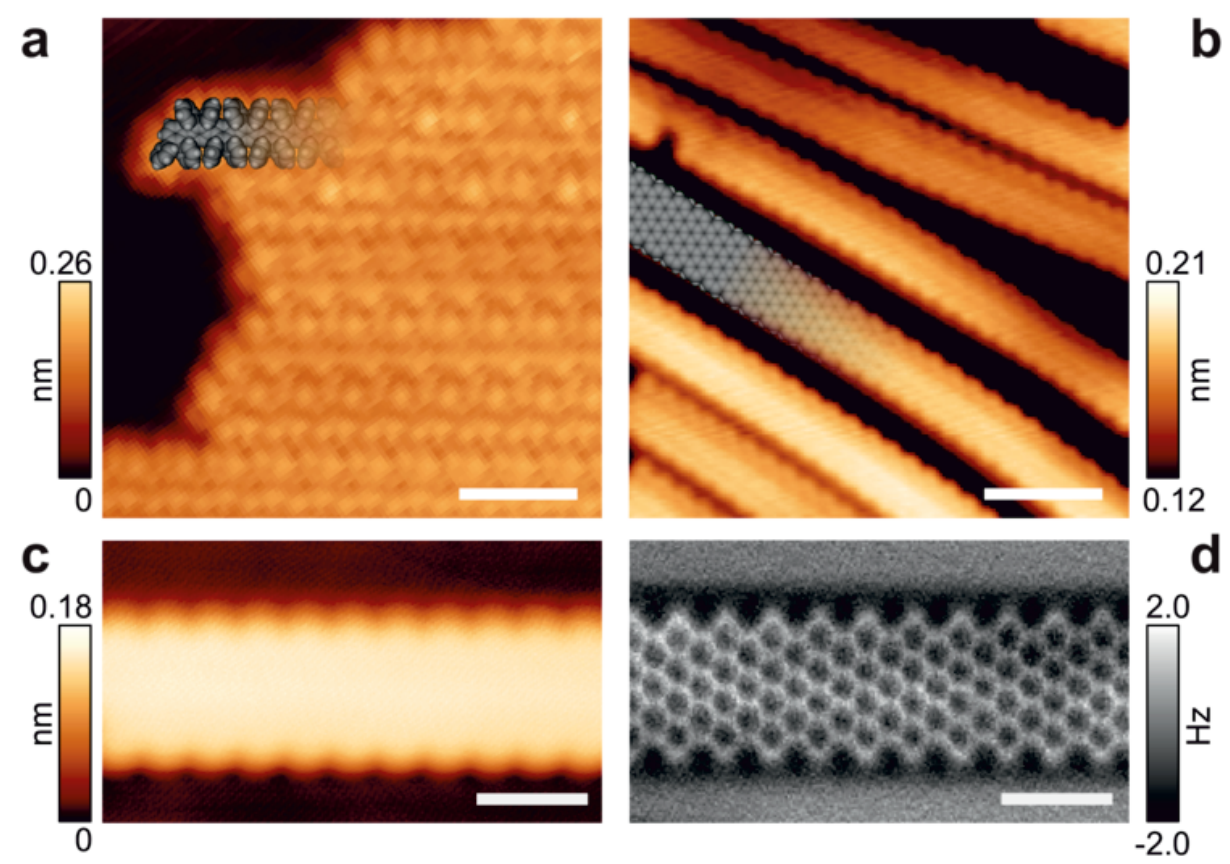

Figure 2. (a) Scanning tunneling microscopy (STM) topography image and structural model of the polymer intermediate 8 formed after annealing to $250^{\circ} \mathrm{C}(V=1.3 \mathrm{~V}, I=0.03 \mathrm{nA}$, Scale bar: $2 \mathrm{~nm}$ ). (b) STM topography image and structural model of 9-AGNRs 9 formed by annealing the polymer sample to $350^{\circ} \mathrm{C}(V=0.1 \mathrm{~V}, I=0.5 \mathrm{nA}$, Scale bar: $2 \mathrm{~nm})$. (c) High-resolution STM topography image of a single 9-AGNR $(V=0.1 \mathrm{~V}, I=0.5 \mathrm{nA}$, Scale bar: $1 \mathrm{~nm})$. (d) Highresolution non-contact atomic force microscopy frequency shift image of 9-AGNR using a COfunctionalized tip with oscillation amplitude of $70 \mathrm{pm}$ (Scale bar: $1 \mathrm{~nm}$ ). 
a

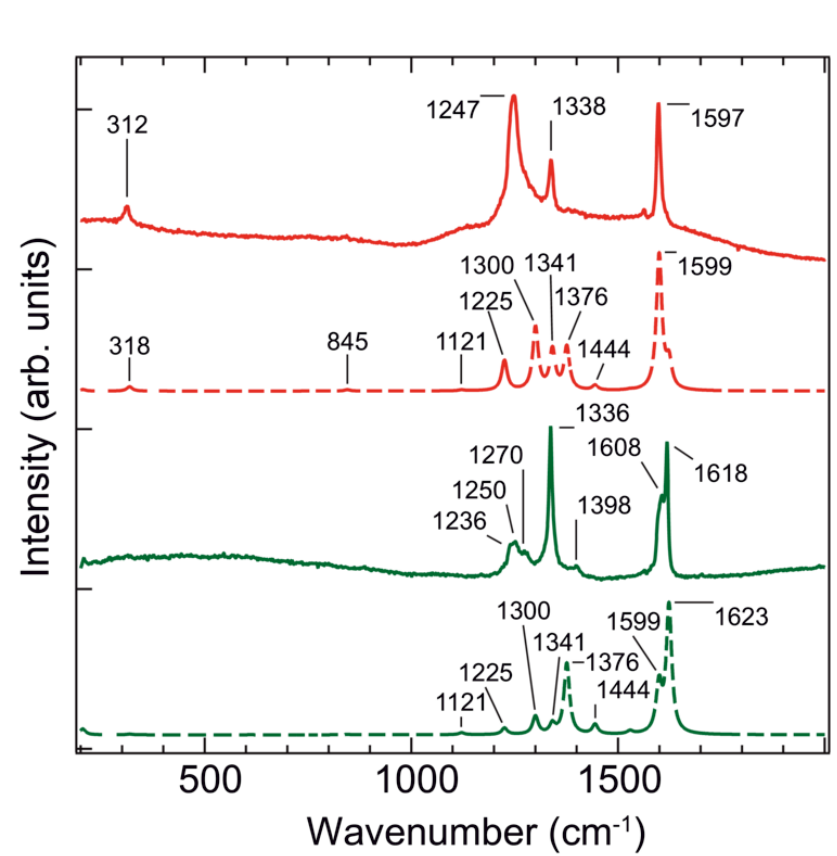

b

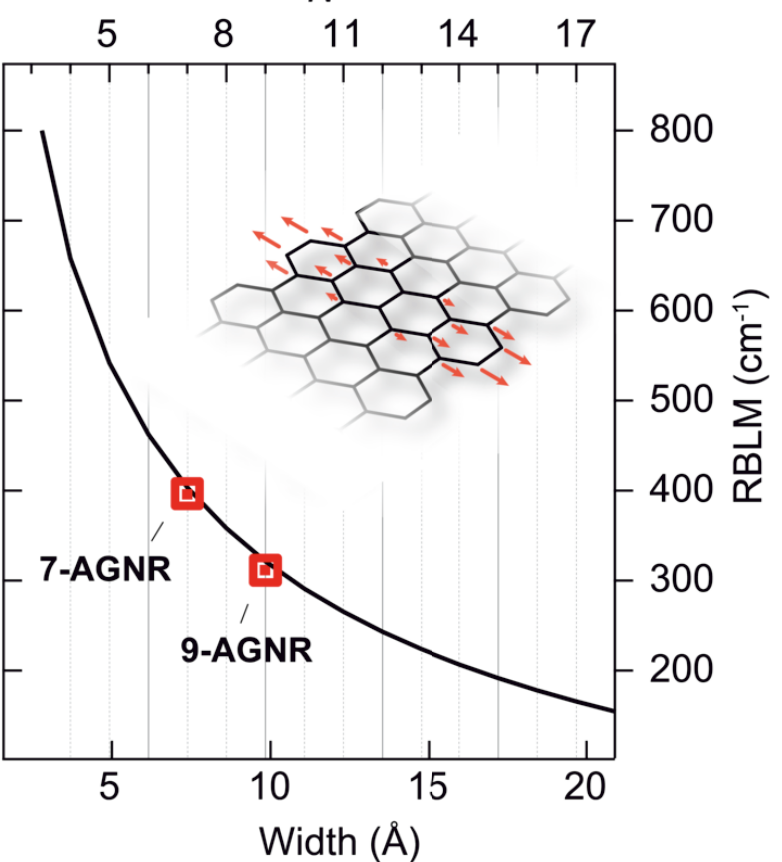

Figure 3. Raman characterization of 9-AGNRs on $\mathrm{Au}(111)$. (a) Experimental Raman spectra at monolayer coverage using $785 \mathrm{~nm}$ (solid red) and $532 \mathrm{~nm}$ (solid green) laser lines as well as DFT-based Raman simulations at corresponding photon energies of $1.58 \mathrm{eV}$ (dashed red) and $2.33 \mathrm{eV}$ (dashed green). (b) Wavenumber of the radial-breathing-like mode (RBLM) for 7AGNRs $^{10}$ and 9-AGNRs, together with predicted ${ }^{51}$ width-dependent RBLM wavenumbers for armchair GNRs. 

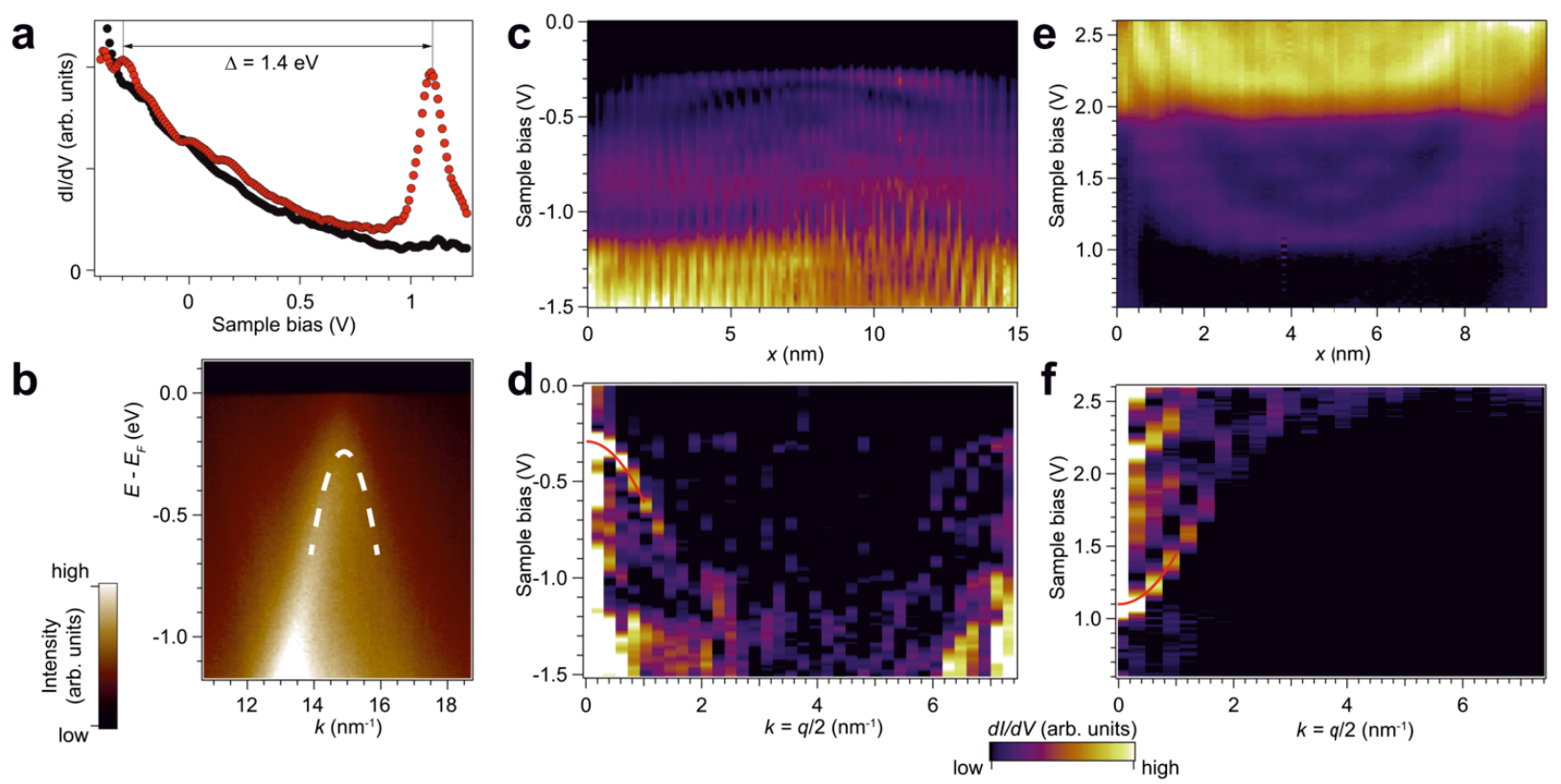

Figure 4. Electronic structure of 9-AGNRs. (a) $d I / d V$ spectra recorded on the edge of a 9-AGNR (red) and on the $\mathrm{Au}(111)$ substrate (black) (tip stabilized at $V=-0.5 \mathrm{~V}, I=10 \mathrm{nA}$ ). Band edges are indicated based on FT-STS data (Table 1). (b) ARPES intensity plot as function of energy $\left(E-E_{F}\right)$ and $k$ vector parallel to the ribbons $(h v=50 \mathrm{eV})$. The white dashed line shows the best fit to the valence band near $k=2 \pi / a=14.8 \mathrm{~nm}^{-1}$. (c) Equidistant $d I / d V$ spectra of occupied states, recorded along the edge of a 9-AGNR of length $35 a$ between the terminus and a defect (stabilization set point $V=-1.5 \mathrm{~V}, I=10 \mathrm{nA}$, spacing $\delta x=0.1 \mathrm{~nm}$ ) (d) Line-by-line Fourier transform of (c) for $0 \leq \frac{q}{2} \leq \frac{\pi}{a}$, including parabolic fit near $\frac{q}{2}=0$. (e) Analogous $d I / d V$ data for the unoccupied states, recorded along the edge of a 9-AGNR of length 23 a between two defects (set point $V=2.6 \mathrm{~V}, I=1 \mathrm{nA}$, spacing $\delta x=0.125 \mathrm{~nm}$ ). (f) Line-by-line Fourier transform of (e). 
a

$$
\underline{\boldsymbol{\nabla}} h=0.1 \mathrm{~nm}
$$

\section{$\mathrm{CB}$}

VB

tis
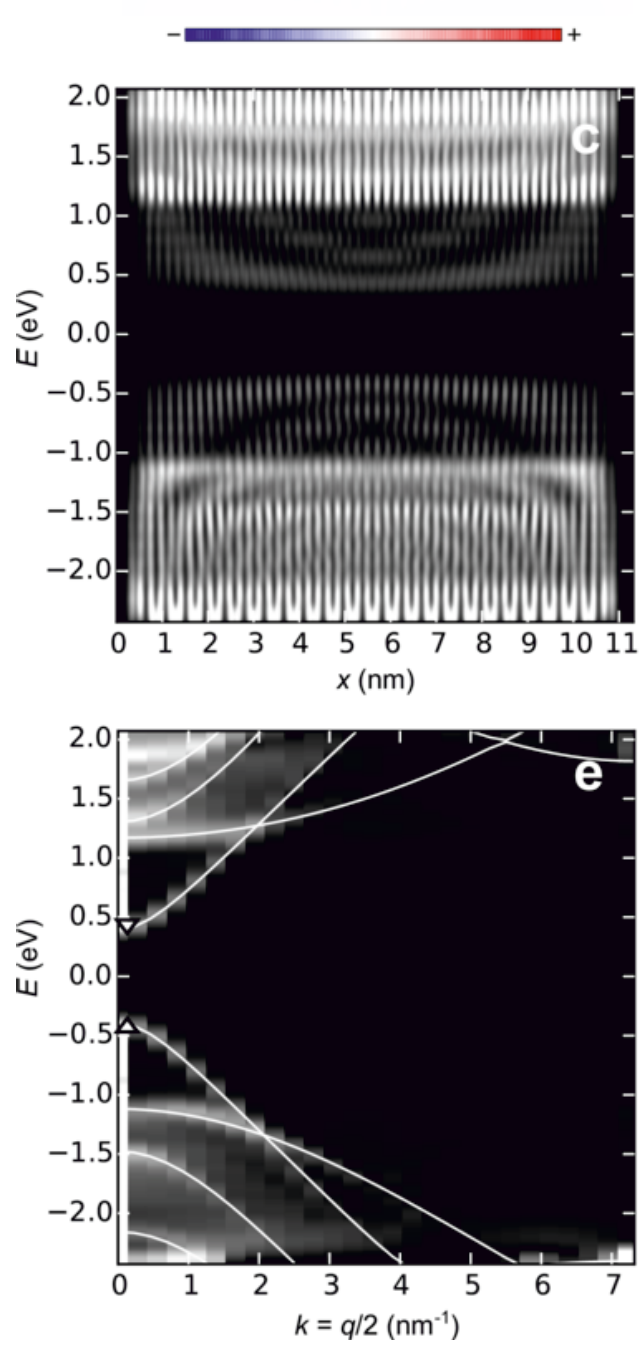

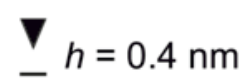

b
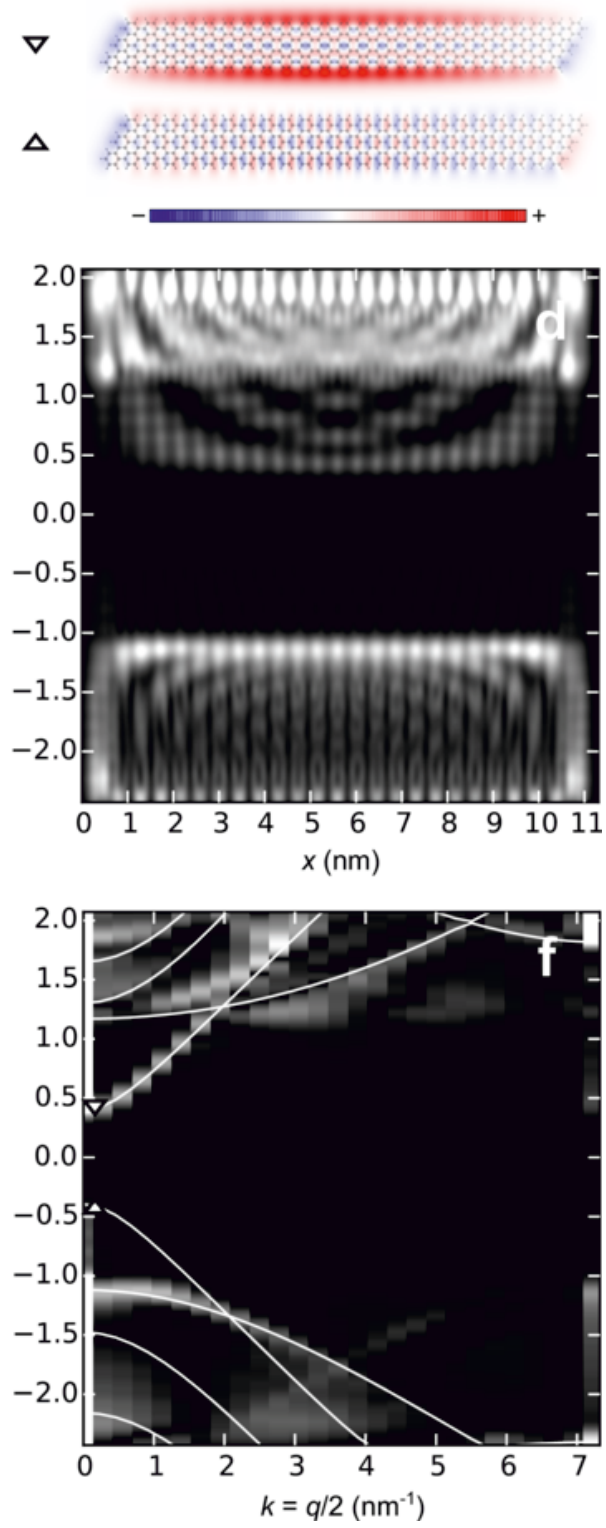

Figure 5. Effect of tip-sample distance on probed local density of states (LDOS). (a),(b) KohnSham orbitals at the band edges of conduction band (CB) and valence band (VB) for 9-AGNR of length $24 a$ evaluated at $0.1 \mathrm{~nm}$ (a) and $0.4 \mathrm{~nm}$ (b) above the GNR. (c), (d) LDOS along the 9AGNR, integrated across the ribbon. The energy zero is chosen as the center of the gap. (e), (f) Fourier-transformed LDOS for $0 \leq k \leq \frac{\pi}{a}$ with bands of infinite 9-AGNR superposed as white lines. 

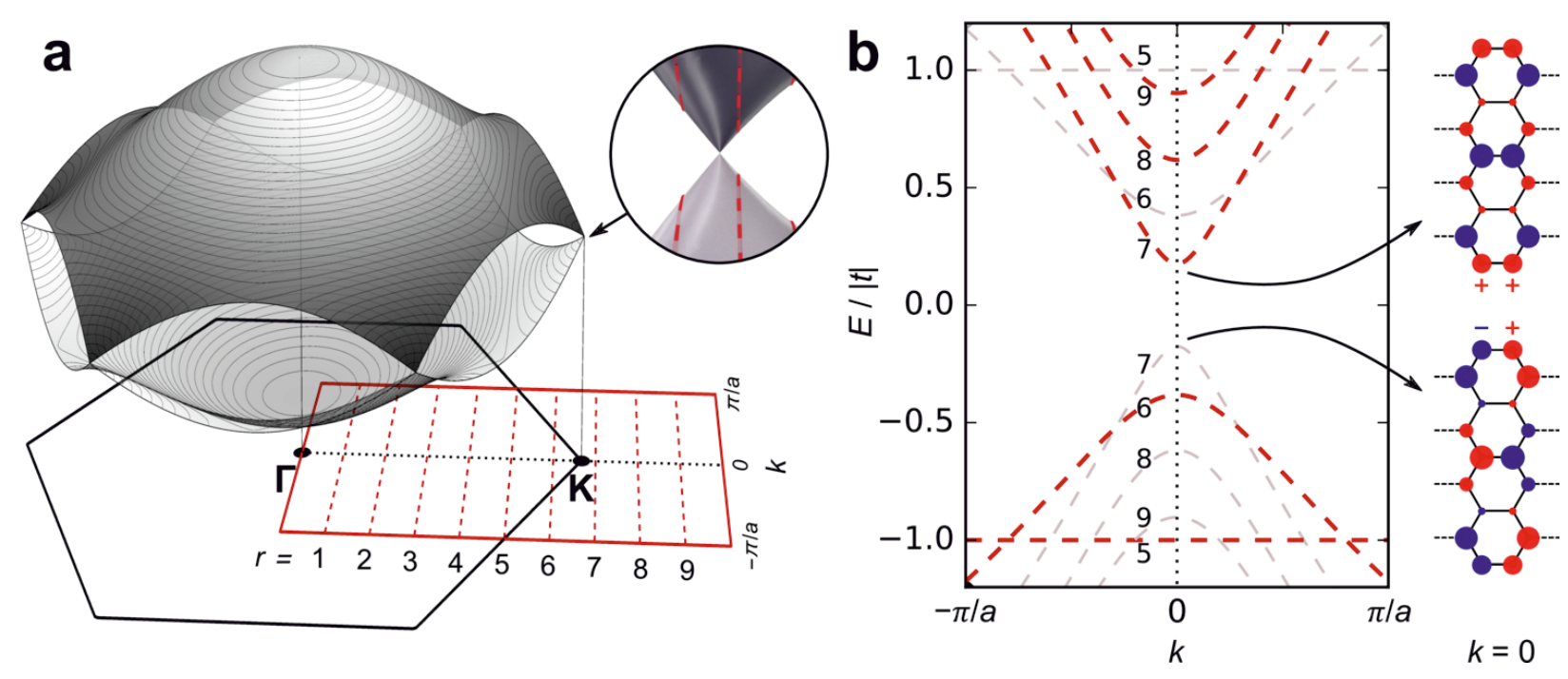

Figure 6. Symmetry of AGNR states in tight binding. (a) First Brillouin zone of graphene (black hexagon) and its $\pi$-band structure (black/grey surfaces) with inset displaying bands near the Dirac cone. Also shown are the first Brillouin zone (red rectangle) and cutting lines (red dashed lines) for infinite length 9-AGNRs. (b) Energy bands corresponding to cutting lines $r=5,6, \ldots$, 9 in (a). At $k=0$, the electronic states of some bands (thick red) do not change sign along the GNR axis, while others (thin gray) change sign once per unit cell, thus making them more difficult to resolve in STS near $k=0$. The wave functions of valence and conduction band are depicted on the right, indicating their weight (circle area) and sign (blue/red color) on each lattice site. 


\begin{tabular}{l|ll|lc} 
& \multicolumn{2}{|c|}{ Valence band } & \multicolumn{2}{c}{ Conduction band } \\
\cline { 2 - 5 } & $m^{*}\left(m_{e}\right)$ & $E_{0}(\mathrm{eV})$ & $m^{*}\left(m_{e}\right)$ & $E_{0}(\mathrm{eV})$ \\
\hline FT-STS & $0.12 \pm 0.03$ & $-0.28 \pm 0.01$ & $0.11 \pm 0.03$ & $1.10 \pm 0.01$ \\
\hline ARPES & $0.09 \pm 0.02$ & $-0.25 \pm 0.04$ & - & - \\
\hline DFT (PBE) & 0.10 & - & 0.10 & - \\
\hline$G_{0} W_{0}(@ P B E)$ & 0.08 & - & 0.08 &
\end{tabular}

Table 1. Band edges and effective masses of valence band (VB) and conduction band (CB) of the 9-AGNR, as obtained from Fourier-transformed scanning tunneling spectroscopy (FT-STS), angle-resolved photoemission spectroscopy (ARPES), density functional theory (DFT) and oneshot $G W\left(G_{0} W_{0}\right)$. Band masses are obtained from two-parameter least squares fits in the range $0 \mathrm{~nm}^{-1} \leq k \leq 1 \mathrm{~nm}^{-1}$

\section{ASSOCIATED CONTENT}

Supporting Information. Includes synthesis steps of precursor monomer, supplementary STM and AFM data of 9-AGNRs, details of Raman and $G W$ calculations, relation between wave function decay and Gaussian filtering, and tight-binding analysis of nodal structure of AGNR wave functions (PDF).

\section{AUTHOR INFORMATION}

\section{Corresponding Author}


* pascal.ruffieux@empa.ch

\section{Present Addresses}

$\dagger$ Department of Physics, University of York, YO10 5DD Heslington, United Kingdom

\section{Author Contributions}

P.R., R.F., X.F., A.N. and K.M. conceived and supervised the experiments. T.D. synthesized the precursor monomers. H.S. developed the on-surface synthesis protocols and performed the STM and STS analysis. S.W. performed nc-AFM imaging. J.R.S. performed Raman spectroscopy. J.L. and N.C.P. performed ARPES under supervision of M.S.. L.L. and V.M. performed Raman calculations. P.S. and C.A.P provided structural models for the polymer on Au(111). L.T. performed electronic structure calculations, STS simulations, AFM simulations and tight-binding analysis. P.R. and L.T. produced the figures. L.T. and P.R. wrote the paper. All authors have commented on and given approval to the final version of the manuscript. $\ddagger$ These authors contributed equally.

\section{Notes}

The authors declare no competing financial interest.

\section{ACKNOWLEDGMENT}

This work was supported by the Swiss National Science Foundation (SNSF), the Office of Naval Research BRC Program, the European Science Foundation (ESF) under the EUROCORES Programme EuroGRAPHENE, the European Commission Graphene Flagship, and by the State Secretariat for Education, Research and Innovation via the COST Action MP0901 NanoTP. L. L. was supported by a Eugene P. Wigner Fellowship at Oak Ridge National Laboratory. 
Computational resources were provided by the Swiss National Supercomputing Centre (CSCS) under project ID s670 and the Center for Computational Innovation at Rensselaer Polytechnic Institute. We thank Aliaksandr Yakutovich for helpful advice concerning AFM simulations.

\section{REFERENCES}

(1) Novoselov, K. S.; Geim, A. K.; Morozov, S. V.; Jiang, D.; Zhang, Y.; Dubonos, S. V.; Grigorieva, I. V.; Firsov, A. A. Electric Field Effect in Atomically Thin Carbon Films. Science 2004, 306, 666-669.

(2) Cheng, R.; Bai, J.; Liao, L.; Zhou, H.; Chen, Y.; Liu, L.; Lin, Y.-C.; Jiang, S.; Huang, Y.; Duan, X. High-Frequency Self-Aligned Graphene Transistors with Transferred Gate Stacks. Proc. Natl. Acad. Sci. U. S. A. 2012, 109, 11588-11592.

(3) Schwierz, F. Graphene Transistors. Nat. Nanotechnol. 2010, 5, 487-496.

(4) Schwierz, F. Graphene Transistors: Status, Prospects, and Problems. Proc. IEEE 2013, $101,1567-1584$.

(5) Barone, V.; Hod, O.; Scuseria, G. E. Electronic Structure and Stability of Semiconducting Graphene Nanoribbons. Nano Lett. 2006, 6, 2748-2754.

(6) Yang, L.; Park, C.-H.; Son, Y.-W.; Cohen, M. L.; Louie, S. G. Quasiparticle Energies and Band Gaps in Graphene Nanoribbons. Phys. Rev. Lett. 2007, 99, 186801.

(7) Gunlycke, D; Areshkin, D. A.; White, C. T. Semiconducting Graphene Nanostrips with Edge Disorder. Appl. Phys. Lett. 2007, 90, 142104.

(8) Stampfer, C.; Güttinger, J.; Hellmüller, S.; Molitor, F.; Ensslin, K.; Ihn, T. Energy Gaps in Etched Graphene Nanoribbons. Phys. Rev. Lett. 2009, 102, 56403.

(9) Ma, L.; Wang, J.; Ding, F. Recent Progress and Challenges in Graphene Nanoribbon Synthesis. ChemPhysChem 2013, 14, 47-54.

(10) Cai, J.; Ruffieux, P.; Jaafar, R.; Bieri, M.; Braun, T.; Blankenburg, S.; Muoth, M.; Seitsonen, A. P.; Saleh, M.; Feng, X.; Müllen, K.; Fasel, R. Atomically Precise Bottom-Up Fabrication of Graphene Nanoribbons. Nature 2010, 466, 470-473.

(11) Lipton-Duffin, J. A.; Ivasenko, O.; Perepichka, D. F.; Rosei, F. Synthesis of Polyphenylene Molecular Wires by Surface-Confined Polymerization. Small 2009, 5, 592-597.

(12) Zhang, H.; Lin, H.; Sun, K.; Chen, L.; Zagranyarski, Y.; Aghdassi, N.; Duhm, S.; Li, Q.; Zhong, D.; Li, Y.; Müllen, K.; Fuchs, H.; Chi, L. On-Surface Synthesis of Rylene-Type Graphene Nanoribbons. J. Am. Chem. Soc. 2015, 137, 4022-4025. 
(13) Chen, Y.-C.; de Oteyza, D. G.; Pedramrazi, Z.; Chen, C.; Fischer, F. R.; Crommie, M. F. Tuning the Band Gap of Graphene Nanoribbons Synthesized from Molecular Precursors. ACS Nano 2013, 7, 6123-6128.

(14) Wassmann, T.; Seitsonen, A. P.; Saitta, A. M.; Lazzeri, M.; Mauri, F. Structure, Stability, Edge States, and Aromaticity of Graphene Ribbons. Phys. Rev. Lett. 2008, 101.

(15) Kimouche, A.; Ervasti, M. M.; Drost, R.; Halonen, S.; Harju, A.; Joensuu, P. M.; Sainio, J.; Liljeroth, P. Ultra-Narrow Metallic Armchair Graphene Nanoribbons. Nat. Commun. 2015, 6, 10177.

(16) Basagni, A.; Sedona, F.; Pignedoli, C. A.; Cattelan, M.; Nicolas, L.; Casarin, M.; Sambi, M. Molecules-Oligomers-Nanowires-Graphene Nanoribbons: A Bottom-Up Stepwise OnSurface Covalent Synthesis Preserving Long-Range Order. J. Am. Chem. Soc. 2015, 137, 18021808.

(17) Fujita, M.; Wakabayashi, K.; Nakada, K.; Kusakabe, K. Peculiar Localized State at Zigzag Graphite Edge. J. Phys. Soc. Jpn. 1996, 65, 1920-1923.

(18) Söde, H.; Talirz, L.; Gröning, O.; Pignedoli, C. A.; Berger, R.; Feng, X.; Müllen, K.; Fasel, R.; Ruffieux, P. Electronic Band Dispersion of Graphene Nanoribbons via FourierTransformed Scanning Tunneling Spectroscopy. Phys. Rev. B 2015, 91, 45429.

(19) Li, G.; Yoon, K.-Y.; Zhong, X.; Zhu, X.; Dong, G. Efficient Bottom-Up Preparation of Graphene Nanoribbons by Mild Suzuki-Miyaura Polymerization of Simple Triaryl Monomers. Chem. - Eur.J. 2016, 22, 9116-9120.

(20) Maddela, S.; Makula, A.; Maddela, R. Synthesis of Isatin-Quinoline Conjugates as Possible Biologically Active Agents. Toxicol. Environ. Chem. 2014, 96, 1-11.

(21) Miljanić, O. Š.; Vollhardt, K. P.; Whitener, G. D. An Alkyne Metathesis-Based Route to Ortho-Dehydrobenzannulenes. Synlett 2002, 0029-0034.

(22) Gross, L.; Mohn, F.; Moll, N.; Liljeroth, P.; Meyer, G. The Chemical Structure of a Molecule Resolved by Atomic Force Microscopy. Science 2009, 325, 1110-1114.

(23) van der Lit, J.; Boneschanscher, M. P.; Vanmaekelbergh, D.; Ijäs, M.; Uppstu, A.; Ervasti, M.; Harju, A.; Liljeroth, P.; Swart, I. Suppression of Electron-Vibron Coupling in Graphene Nanoribbons Contacted via a Single Atom. Nat. Commun. 2013, 4, 2023.

(24) Talirz, L.; Söde, H.; Cai, J.; Ruffieux, P.; Blankenburg, S.; Jafaar, R.; Berger, R.; Feng, X.; Müllen, K.; Passerone, D.; Fasel, R.; Pignedoli, C. A. Termini of Bottom-Up Fabricated Graphene Nanoribbons. J. Am. Chem. Soc. 2013, 135, 2060-2063.

(25) Sanders, G. D.; Nugraha, A. R. T.; Saito, R.; Stanton, C. J. Coherent Radial-Breathinglike Phonons in Graphene Nanoribbons. Phys. Rev. B 2012, 85, 205401.

(26) Denk, R.; Hohage, M.; Zeppenfeld, P.; Cai, J.; Pignedoli, C. A.; Söde, H.; Fasel, R.; Feng, X.; Müllen, K.; Wang, S.; Prezzi, D.; Ferretti, A.; Ruini, A.; Molinari, E.; Ruffieux, P. 
Exciton-Dominated Optical Response of Ultra-Narrow Graphene Nanoribbons. Nat. Commun. 2014, 5, 4253 .

(27) Prezzi, D.; Varsano, D.; Ruini, A.; Marini, A.; Molinari, E. Optical Properties of Graphene Nanoribbons: The Role of Many-Body Effects. Phys. Rev. B 2008, 77, 41404.

(28) Wang, S.; Wang, J. Quasiparticle Energies and Optical Excitations in Chevron-Type Graphene Nanoribbon. J. Phys. Chem. C 2012, 116, 10193-10197.

(29) Krukau, A. V.; Vydrov, O. A.; Izmaylov, A. F.; Scuseria, G. E. Influence of the Exchange Screening Parameter on the Performance of Screened Hybrid Functionals. J. Chem. Phys. 2006, 125, 224106.

(30) Linden, S.; Zhong, D.; Timmer, A.; Aghdassi, N.; Franke, J. H.; Zhang, H.; Feng, X.; Müllen, K.; Fuchs, H.; Chi, L.; Zacharias, H. Electronic Structure of Spatially Aligned Graphene Nanoribbons on Au(788). Phys. Rev. Lett. 2012, 108, 216801.

(31) Ruffieux, P.; Cai, J.; Plumb, N. C.; Patthey, L.; Prezzi, D.; Ferretti, A.; Molinari, E.; Feng, X.; Müllen, K.; Pignedoli, C. A.; Fasel, R. Electronic Structure of Atomically Precise Graphene Nanoribbons. ACS Nano 2012, 6, 6930-6935.

(32) Tersoff, J. D.; Hamann, D. R. Theory of the Scanning Tunneling Microscope. Phys. Rev. $B$ 1985, 31, 805-813.

(33) Bennett, P. B.; Pedramrazi, Z.; Madani, A.; Chen, Y.-C.; de Oteyza, D. G.; Chen, C.; Fischer, F. R.; Crommie, M. F.; Bokor, J. Bottom-up Graphene Nanoribbon Field-Effect Transistors. Appl. Phys. Lett. 2013, 103, 253114.

(34) Perdew, J. P.; Burke, K.; Ernzerhof, M. Generalized Gradient Approximation Made Simple. Phys. Rev. Lett. 1996, 77, 3865.

(35) Trevisanutto, P. E.; Giorgetti, C.; Reining, L.; Ladisa, M.; Olevano, V. Ab Initio GW Many-Body Effects in Graphene. Phys. Rev. Lett. 2008, 101, 226405.

(36) Koch, M.; Ample, F.; Joachim, C.; Grill, L. Voltage-Dependent Conductance of a Single Graphene Nanoribbon. Nat. Nanotechnol. 2012, 7, 713-717.

(37) Wakabayashi, K.; Sasaki, K.; Nakanishi, T.; Enoki, T. Electronic States of Graphene Nanoribbons and Analytical Solutions. Sci. Technol. Adv. Mater. 2010, 11, 54504.

(38) Nakada, K.; Fujita, M.; Dresselhaus, G.; Dresselhaus, M. S. Edge State in Graphene Ribbons: Nanometer Size Effect and Edge Shape Dependence. Phys. Rev. B 1996, 54, 17954 17961.

(39) Giessibl, F. J. Atomic Resolution on $\mathrm{Si}(111)-(7 \times 7)$ by Noncontact Atomic Force Microscopy with a Force Sensor Based on a Quartz Tuning Fork. Appl. Phys. Lett. 2000, 76, 1470-1472.

(40) The CP2K developers group, 2013. CP2K is freely available from http://www.cp2k.org (accessed Jun 20, 2013). 
(41) Tersoff, J. Method for the Calculation of Scanning Tunneling Microscope Images and Spectra. Phys. Rev. B 1989, 40, 11990.

(42) Giannozzi, P.; Baroni, S.; Bonini, N.; Calandra, M.; Car, R.; Cavazzoni, C.; Davide Ceresoli; Chiarotti, G. L.; Cococcioni, M.; Dabo, I.; Corso, A. D.; Gironcoli, S. de; Fabris, S.; Fratesi, G.; Gebauer, R.; Gerstmann, U.; Gougoussis, C.; Anton Kokalj; Lazzeri, M.; MartinSamos, L.; et al. QUANTUM ESPRESSO: A Modular and Open-Source Software Project for Quantum Simulations of Materials. J. Phys.: Condens. Matter 2009, 21, 395502.

(43) Hamann, D. R. Optimized Norm-Conserving Vanderbilt Pseudopotentials. Phys. Rev. B 2013, 88,85117 .

(44) Schlipf, M.; Gygi, F. Optimization Algorithm for the Generation of ONCV Pseudopotentials. Comput. Phys. Commun. 2015, 196, 36-44.

(45) Kresse, G.; Furthmüller, J. Efficiency of Ab-Initio Total Energy Calculations for Metals and Semiconductors Using a Plane-Wave Basis Set. Comput. Mater. Sci. 1996, 6, 15-50.

(46) PHONON Software, version 6.15; www.computingformaterials.com.

(47) Hybertsen, M. S.; Louie, S. G. Electron Correlation in Semiconductors and Insulators: Band Gaps and Quasiparticle Energies. Phys. Rev. B 1986, 34, 5390-5413.

(48) Deslippe, J.; Samsonidze, G.; Strubbe, D. A.; Jain, M.; Cohen, M. L.; Louie, S. G. BerkeleyGW: A Massively Parallel Computer Package for the Calculation of the Quasiparticle and Optical Properties of Materials and Nanostructures. Comput. Phys. Commun. 2012, 183, 1269-1289.

(49) Rozzi, C. A.; Varsano, D.; Marini, A.; Gross, E. K. U.; Rubio, A. Exact Coulomb Cutoff Technique for Supercell Calculations. Phys. Rev. B 2006, 73, 205119.

(50) Deslippe, J.; Samsonidze, G.; Jain, M.; Cohen, M. L.; Louie, S. G. Coulomb-Hole Summations and Energies for GW Calculations with Limited Number of Empty Orbitals: A Modified Static Remainder Approach. Phys. Rev. B 2013, 87, 165124.

(51) Vandescuren, M.; Hermet, P.; Meunier, V.; Henrard, L.; Lambin, P. Theoretical Study of the Vibrational Edge Modes in Graphene Nanoribbons. Phys. Rev. B 2008, 78, 195401. 
SYNOPSIS

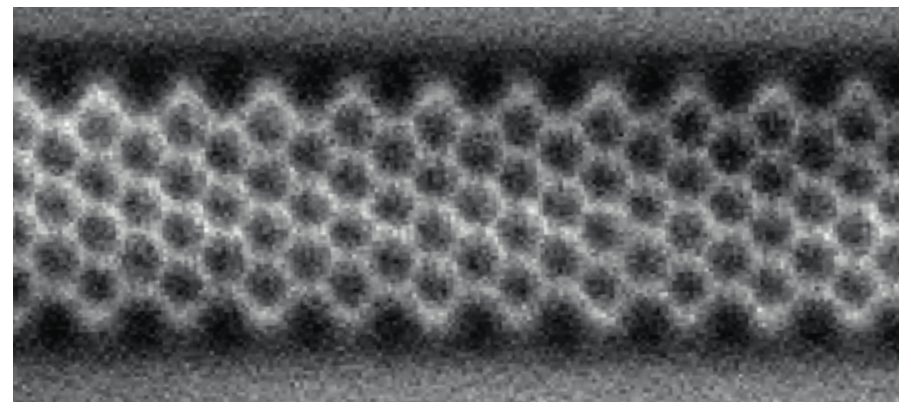

